\title{
Análisis de controversias en reconstrucción mamaria con colgajo DIEP
}

\section{Analysis of debated uses in mammary reconstruction with DIEP flap}

\author{
Casado Sánchez, C.*, Cabrera Sánchez, E.**, Redondo Camacho, A.**, Rioja Torrejón, L. F.***
}

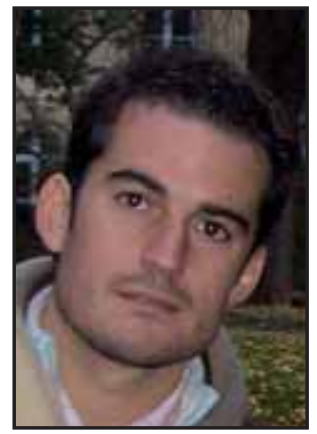

Casado Sánchez, C.

\section{Resumen}

La reconstrucción mamaria mediante colgajo DIEP (Deep Inferior Epigastric Perforator) se encuentra condicionada en muchas ocasiones por características de la paciente o del tratamiento oncológico recibido, de tal forma que diversos grupos de trabajo contraindican este método en caso de existir alguno de esos factores. Nosotros hemos realizado un estudio de los mismos, analizando su impacto sobre la viabilidad del colgajo y su correlación con las complicaciones postoperatorias, para optimizar así su indicación.

Los condicionantes analizados son el tabaco, las intervenciones quirúrgicas previas que afecten en alguna medida la pared abdominal, el sobrepeso u obesidad de la paciente, el volumen de la mama contralateral, el tratamiento radioterápico anterior o posterior a la reconstrucción, y finalmente el gasto sanitario que supone. Estas controversias son a su vez las más discutidas en la literatura al respecto.

Se valoran los resultados obtenidos tras 55 reconstrucciones mamarias mediante colgajo DIEP entre enero de 2000 y noviembre de 2005, así como los estudios y publicaciones más recientes existentes sobre esta materia.

Hemos encontrado una pobre correlación entre los índices de fracaso del colgajo y las controversias estudiadas, determinando así dos contraindicaciones absolutas para que una paciente pudiese beneficiarse de un colgajo DIEP: abdominoplastia previa y radioterapia adyuvante tras reconstrucción inmediata. Los excelentes resultados que se obtienen globalmente, nos llevan a recomendar este colgajo por encima de otras opciones terapéuticas.

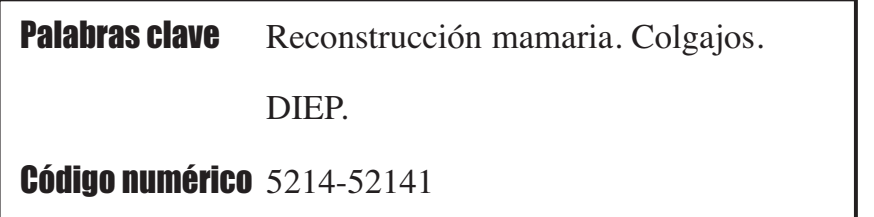

The use of DIEP (Deep Inferior Epigastric Perforator) flap surgery in breast reconstruction is often conditioned by the patient and the oncologic treatment characteristics to such an extent that several working groups advise against this surgical procedure when any of these factors is present. In the study of this interaction, an analysis of its impact on the flap feasibility and its correlation with postoperative complications was carried out as an attempt to gain accuracy in the prescription of this technique.

The factors under assessment were smoking habits, previous surgical interventions involving the abdominal wall to any extent, patient obesity, contralateral breast size, pre- and post-reconstruction radiotherapy, and lastly the medical costs. These factors coincide with the most debated issues in the related literature.

The results of 55 breast reconstructions by DIEP flap surgery performed between january 2000 and november 2005 were evaluated, together with the latest studies and publications on the subject.

The study revealed a poor correlation between DIEP flap surgery failure rates and the conditioning factors analyzed, while two scenarios proved to be absolutely incompatible with successful DIEP flap surgery: previous abdominoplasty and adjuvant radiotherapy after immediate reconstruction. In light of the excellent global results obtained, DIEP flap surgery is recommended over other therapeutic options.

$\begin{array}{ll}\text { Key words } & \text { Mammary reconstruction. Flaps. DIEP. } \\ \text { Numeral Gode } \quad 5214-52141\end{array}$

\footnotetext{
* Médico Interno Residente.

* Médico Adjunto.

* J Jefe del Servicio.
}

Servicio de Cinugía Plástica y Reparadora. Hospital Universitario Reina Sofía. Córdoba. España. 


\section{Introducción}

La reconstrucción mamaria mediante colgajos libres abdominales ha sufrido una notable evolución desde su descripción, con múltiples modificaciones y variaciones técnicas que han permitido optimizar la indicación de esta interesante opción reconstructiva y han posibilitado el desarrollo de otros métodos menos agresivos respecto a las zonas donantes.

Diseñado y aplicado clínicamente por primera vez por Koshima y Soeda para el tratamiento de defectos de la cavidad oral, el colgajo DIEP (Deep Inferior Epigastric Perforator) fue empleado como colgajo para reconstrucción mamaria en 1990 por Allen y Treece, popularizándose en la siguiente década.

Desde entonces, los colgajos de perforantes de la epigástrica inferior profunda han pasado a ocupar un lugar puntero en la reconstrucción de la mama, debido al empleo de tejidos autólogos que proporcionan resultados estables y duraderos en el tiempo, con características que pueden llegar a ser excelentes en términos de textura, volumen, ptosis y simetría, añadiendo a ésto una mínima morbilidad del área donante. Estas características favorecen que tanto los cirujanos plásticos como la opinión pública prestigien esta opción reconstructiva, más aún en el contexto actual de una "epidemia" de cáncer de mama.

Son frecuentes en la práctica clínica diaria las ocasiones en las que una paciente, que podría beneficiarse de este colgajo libre, es tratada mediante otras técnicas debido a la existencia de múltiples controversias que llevan a desaconsejar el DIEP, tales como cirugías previas, tratamientos adyuvantes, hábitos de vida o características propias de la paciente, entre otras.

El propósito de nuestro trabajo es abordar aquellos factores que condicionan la contraindicación de reconstrucción mamaria mediante colgajo DIEP, establecer su correlación con diferentes complicaciones postoperatorias, y así optimizar su indicación. Basándonos en nuestra experiencia, hemos desarrollado una sólida línea de trabajo y una serie de recomendaciones, que nos llevan a definir el DIEP como un método reconstructivo difícilmente superable por otras opciones.

\section{Material y método}

Presentamos una serie de 55 reconstrucciones mamarias mediante colgajo DIEP realizadas entre enero de 2000 y noviembre de 2005 en la Unidad de Reconstrucción Microquirúrgica del Servicio de Cirugía Plástica y Reparadora del Hospital Reina Sofía de Córdoba (España).
La media de edad de nuestras pacientes fue de 50 años; todas ellas habían sido sometidas a mastectomía y en todas se practicó reconstrucción mamaria diferida mediante colgajo DIEP. La media de tiempo transcurrido entre la mastectomía y la reconstrucción fue de 25, 6 meses (moda de 24 meses).

No nos detendremos en describir la anatomía, fundamentos y técnica quirúrgica del colgajo empleado, dado que además de conocidos no son el objeto de nuestro trabajo. El posicionamiento del colgajo, los refinamientos técnicos del mismo y los procedimientos seguidos en el segundo tiempo quirúrgico, ya los hemos descrito en publicaciones previas (1).

Valoramos los factores tradicionalmente más discutidos en la literatura y en los diversos foros científicos sobre reconstrucción mamaria mediante colgajos libres, por su implicación en la incidencia de complicaciones postoperatorias del tipo necrosis grasa, congestión venosa, trombosis arterial y retraso cicatricial, a saber: influencia del tabaco, de las intervenciones quirúrgicas abdominales previas, de la obesidad, del volumen de la mama contralateral, de la radioterapia postmastectomía y del gasto hospitalario.

Entre nuestras 55 pacientes analizadas.

-Tabaco: 15 pacientes eran fumadoras y 40 no fumadoras.

-Cirugías abdominales previas: 14 pacientes habían sido sometidas a intervenciones quirúrgicas previas en el área abdominal. De ellas 9 presentaban cicatrices tipo McBurney; 2, incisiones en hipocondrio que en ningún caso condicionaban la ejecución del colgajo; 2, incisiones tipo Pfannestiel por cesárea y 1 cicatriz media infraumbilical por urgencia ginecológica (embarazo ectópico).

-Obesidad: el índice de masa corporal (IMC) en nuestro grupo presentó una media de $26,9 \mathrm{Kg} / \mathrm{m}^{2}$, es decir, eran pacientes relativamente jóvenes con un sobrepeso discreto.

-Volumen mamario contralateral: en nuestro grupo, siempre tratamos la mama contralateral en un segundo tiempo quirúrgico y en función de las necesidades de la paciente. Cinco necesitaron aumento del volumen de la mama sana; 24 fueron sometidas a mamoplastia de reducción contralateral; se realizaron 16 mastopexias, en su mayoría acompañadas de colocación de prótesis de silicona; finalmente, 6 de las pacientes no requirieron o no aceptaron simetrización mamaria. Cuatro pacientes presentaban mastectomía bilateral: 3 de ellas se reconstruyeron mediante expansor mamario y prótesis de silicona y 1 fue reconstruida mediante colgajo libre bilateral. La reconstrucción teoloareolar se hizo también siempre en un segundo tiempo quirúrgico, mediante colgajos locales para el pezón y tatuaje para la areola (Fig. 1 y 2). 

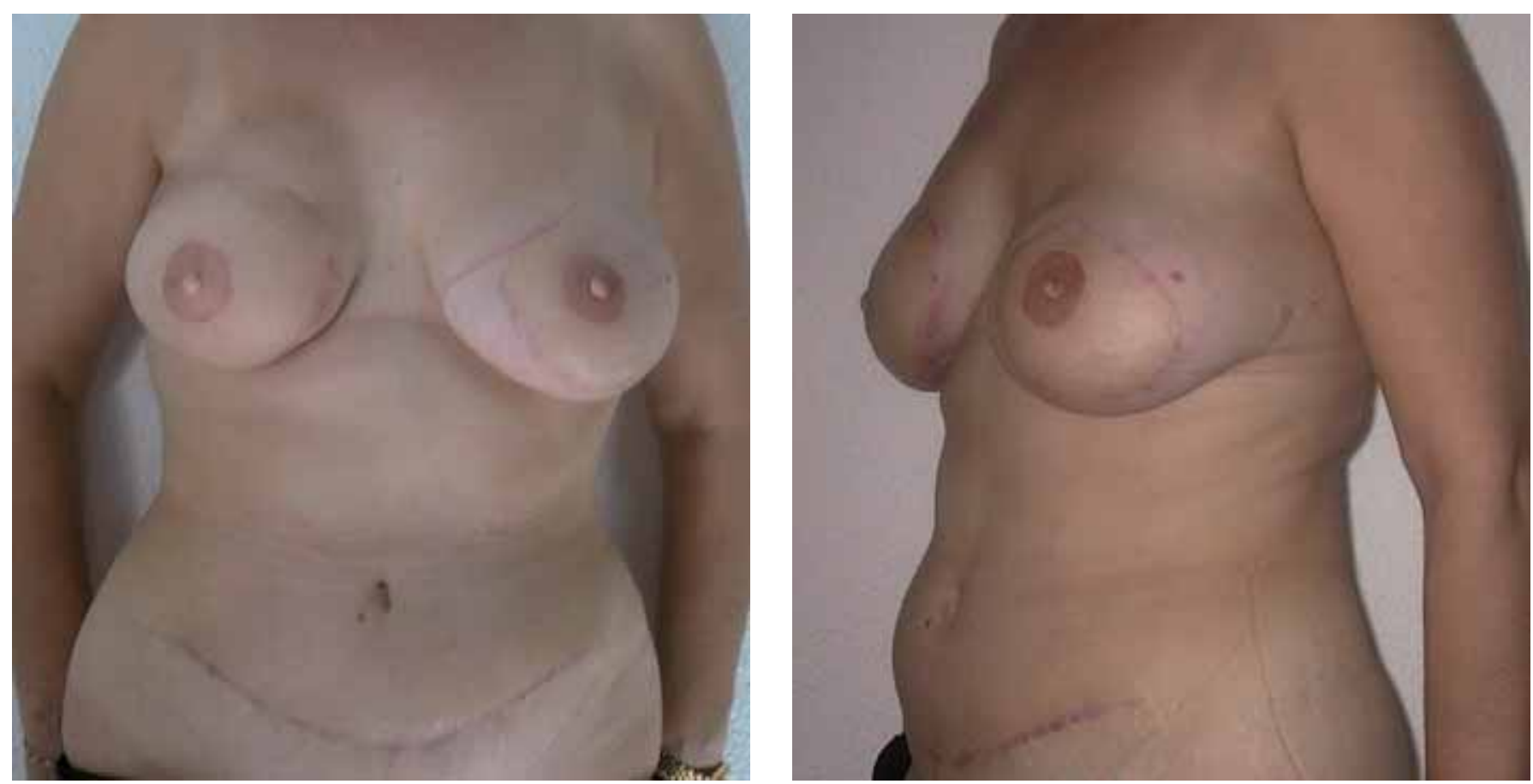

Fig. 1. Reconstrucción mamaria bilateral.: Colgajos DIEP más reconstrucción teloareolar en segundo tiempo quirúrgico. Imagen postoperatoria al año.

-Radioterapia postmastectomía: 43 pacientes habían requerido tratamiento adyuvante con radioterapia tras la mastectomía, de forma previa a la reconstrucción.

-Gasto sanitario.

\section{Resultados}

Tras una serie de 55 reconstrucciones mamarias con DIEP, hemos registrado únicamente 1 caso de necrosis completa del colgajo (esto supone un 1,82\% de los casos) y 3 casos de necrosis parcial $(5,45 \%$ de los casos).

La etiología de la necrosis completa del DIEP radicó en una congestión venosa (Fig. 3). Las características de esta paciente eran las siguientes: paciente de 42 años de edad, sometida a reconstrucción mamaria a los 24 meses de la mastectomía, no fumadora, con leve sobrepeso (IMC $27,1 \mathrm{Kg} / \mathrm{m}^{2}$ ) y sometida en el pasado a una laparotomía exploradora baja por emba-
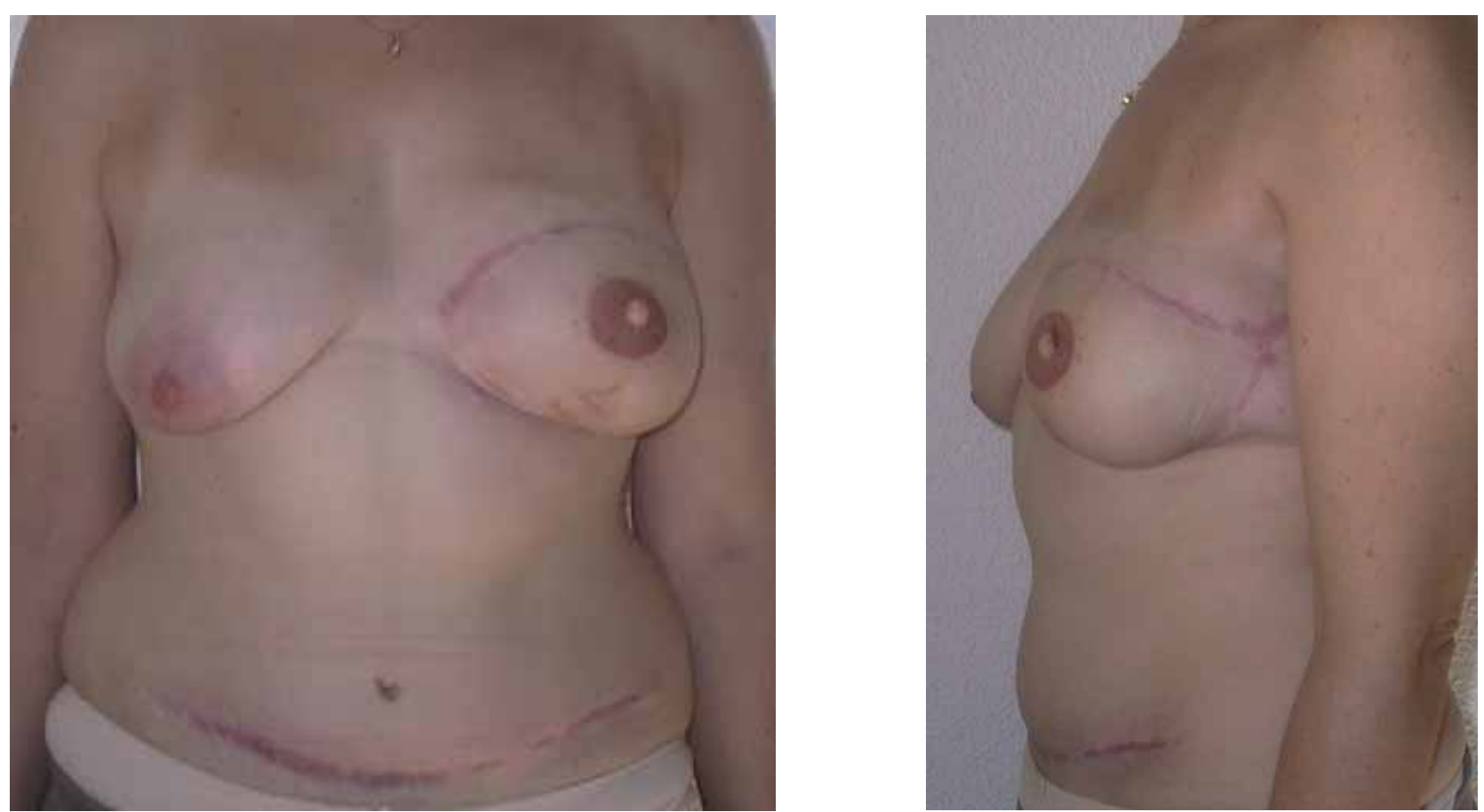

Fig. 2. Reconstrucción mamaria unilateral con colgajo DIEP pendiente de simetrización mamaria contralateral. 


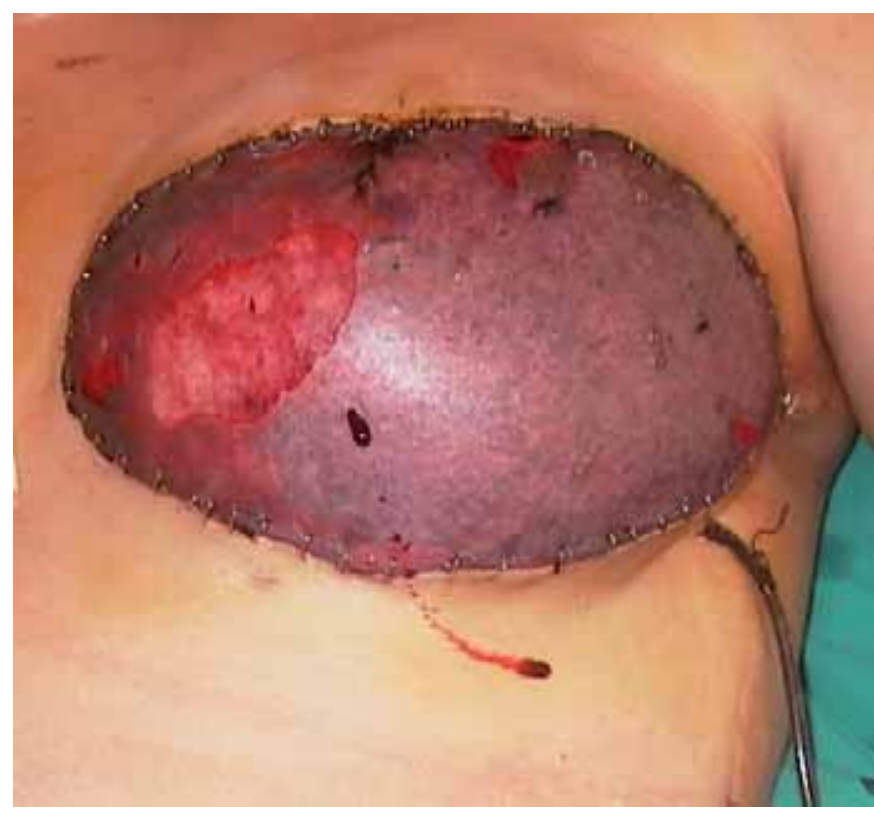

Fig. 3. Necrosis total de colgajo DIEP en postoperatorio inmediato.

razo ectópico. Presentaba mastectomía radical contralateral reconstruida mediante expansor mamario. Se procedió al desbridamiento amplio del colgajo y, dada la ausencia de ambos pedículos toracodorsales que impedía considerar el colgajo de dorsal ancho como opción reconstructiva, ya fuera de forma libre o pediculado, se optó por la cobertura del área cruenta mediante injerto de piel parcial, para posterior reconstrucción mediante expansor tisular.

Respecto a los 3 casos de necrosis parcial del colgajo, 2 se trataron de forma conservadora sin precisar reintervención y, la más significativa, sufrió la pérdida del polo superior de la mama reconstruida. En este caso, se trataba de una paciente no fumadora, sin intervenciones abdominales previas, con IMC de $27,66 \mathrm{Kg} / \mathrm{m}^{2}$, que fue sometida a reconstrucción a los 36 meses de la mastectomía, tras la cual había precisado radioterapia adyuvante. Esta paciente sufrió recidiva tumoral y metástasis a distancia a los 2 años de la reconstrucción.

Como otras complicaciones referimos, 4 revisiones de la microsutura en el postoperatorio por sufrimiento del colgajo, una de las cuales precisó reanastomosis arterial al pedículo toracodorsal ipsilateral (paciente fumadora de 37 años de edad), 2 dehiscencias de sutura a nivel del colgajo sin complicaciones añadidas, y 5 dehiscencias a nivel del territorio donante abdominal ( 2 de los casos en pacientes fumadoras), que se resolvieron mediante curas locales o revisión directa de la sutura.

Creemos que la experiencia de nuestro Servicio en complicaciones postoperatorias, fundamentalmente las referidas a necrosis total y parcial del colgajo, nos indica la ausencia de correlación entre éstas y los factores que inicialmente valoramos en el presente estu- dio. De esta forma hemos conseguido alcanzar unos resultados acordes a las expectativas que ofrece dicho colgajo y hemos establecido una dinámica de trabajo de reconstrucción mamaria que nos permite no obviar el colgajo DIEP en la mayoría de las pacientes mastectomizadas, exceptuando en aquellas a las que haremos referencia más adelante.

\section{Discusión}

Describiremos la relación de factores que suponen una controversia a la hora de indicar o no el colgajo DIEP en las pacientes afectadas, y nuestra actitud respecto a ellos.

\section{Tabaco}

Los efectos vasculares del tabaco son sobradamente conocidos. Podríamos resumirlos en un incremento en los índices de trombosis a través del aumento de los niveles de fibrinógeno plasmático, mayor tendencia a la vasoconstricción dado el descenso de la síntesis del óxido nítrico y así, una prevalencia superior de ateroesclerosis respecto a los no fumadores.

Pese a que distintos estudios recomiendan desaconsejar la reconstrucción mamaria mediante colgajos libres en pacientes fumadoras (2), nosotros no hemos constatado morbilidad adicional en estas pacientes en la serie analizada. Los estudios publicados que hemos citado, no encuentran un incremento en las tasas de complicaciones tempranas, pero sí refieren diferencias estadísticamente significativas en términos de necrosis grasa (13'5\% frente a un 3\% en no fumadoras) y de retraso en la cicatrización (9\% frente a un $3 \%$ en no fumadoras).

Nuestro grupo de trabajo ha obtenido resultados que constatan que la incidencia de trombosis vascular y pérdida del colgajo es similar entre fumadoras y no fumadoras, al igual que otros autores como Nahabedian que tampoco han demostrado morbilidad adicional (3-5).

\section{Intervenciones quirúrgicas abdominales previas}

Las cirugías abdominales son causa común de escepticismo entre los cirujanos plásticos cuando se decide optar por un colgajo abdominal para la reconstrucción. A la posibilidad de que se hubiesen dañado los pedículos vasculares del colgajo, hay que añadir la dificultad que supone evitar la secuela antiestética cicatricial en la mama reconstruída.

El examen físico previo es esencial en toda paciente en la que se plantea la reconstrucción con DIEP, más aún en aquellas en las que se duda de la viabilidad de sus perforantes, caso de las mujeres intervenidas quirúrgicamente. Nosotros continuamos emplean- 


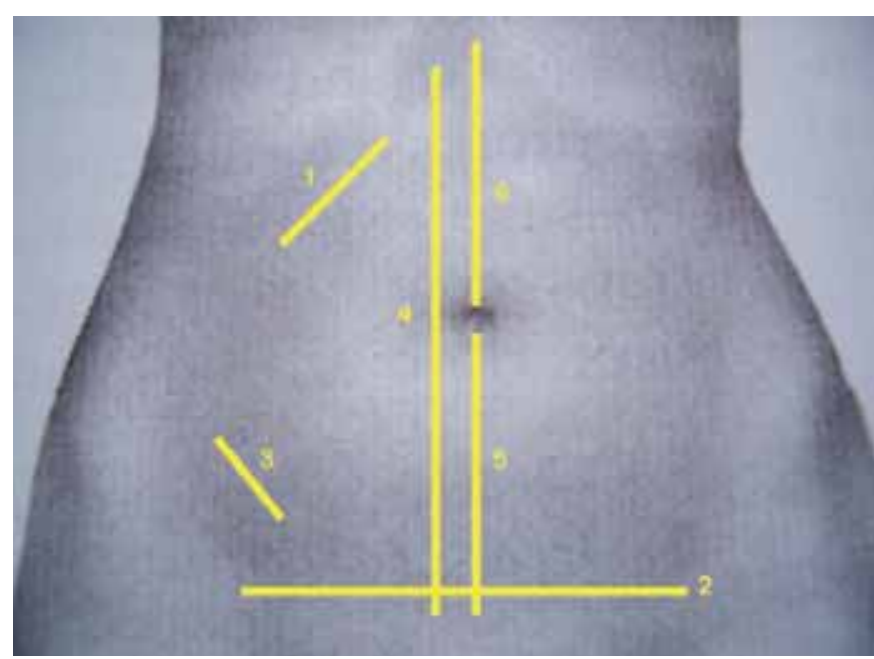

Fig. 4. 1. Incisión de Kocher: bajo el borde costal derecho. 2. Incisión de Pfannestiel: convexa por encima de la sínfisis del pubis. 3. Incisión de McBurney: sobre la espina iliaca anterosuperior. 4. Incisión paramedial derecha: paralela a la línea alba. 5. Incisión media inferior: desde ombligo hasta pubis. 6 . Incisión media superior: desde ombligo hasta apófisis xifoides.

do el ecodopler para valorar la existencia, previa a la reconstrucción, de perforantes vasculares que permitan desarrollar está técnica quirúrgica con preservación muscular. Pero nuestra actitud definitiva respecto a ellas, sigue contemplando una decisión intraoperatoria, basando la elección del pedículo en su observación directa.

Las intervenciones quirúrgicas abdominales más frecuentes tienen su vía de abordaje en las reflejadas en la Figura 4. Según la precisión del cirujano que las realice, y su manejo de los tejidos más o menos cuidadoso, pueden suponer o no una contraindicación.

La vía actual de abordaje en las cesáreas (Pfannestiel) no supone una contraindicación, ya que la arteria epigástrica superior profunda probablemente se encuentre dilatada dada la sección previa de la inferior, y las perforantes serían así de un calibre suficiente. Los abordajes de apendicectomía (McBurney) y de vías biliares (Kocher) no afectarían a los pedículos dominantes localizados más frecuentemente en el territorio periumbilical, además de respetar el territorio vascular derecho. Por esta razón, los abordajes a través de la línea media, condicionarían solo parcialmente la contraindicación del colgajo DIEP (decisión pendiente de la búsqueda prequirúrgica de las perforantes), en función de la experiencia y habilidad del cirujano general para respetar los tejidos superficiales.

La única intervención quirúrgica que supone una contraindicación absoluta es que la paciente se hubiese sometido a una abdominoplastia, dada la sección de las perforantes musculares que se practica en el despegamiento dermograso. Querríamos hacer un especial énfasis en la valoración cuidadosa de las pacientes sometidas a una liposucción abdominal; en el caso de cumplirse esta característica, el cirujano

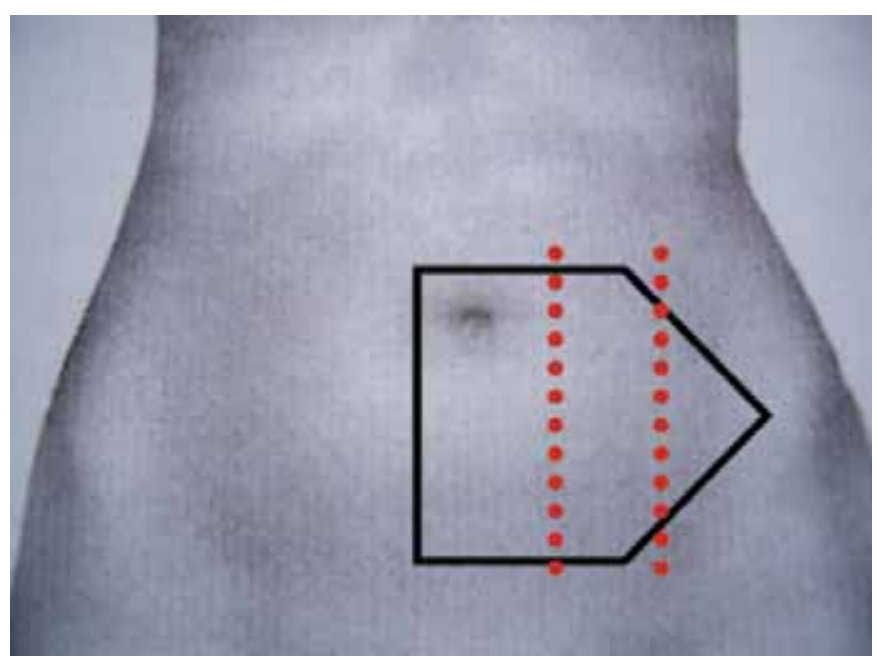

Fig. 5. Sobre el diseño prequirúrgico del colgajo, se esquematizan las dos hileras de perforantes descritas en cada hemiabdomen.

plástico debería extremar las precauciones en el diseño preoperatorio del DIEP, realizando una búsqueda más exhaustiva aún de los potenciales pedículos del futuro colgajo. No podemos obviar el riesgo de lesión vascular que la liposucción entraña en los tejidos superficiales del abdomen de la paciente y, por esta razón, en nuestro Servicio consideramos este tipo de intervención como una contraindicación relativa.

En cualquier caso, se debe siempre contemplar ante cualquier duda sobre la viabilidad vascular, la transformación intraoperatoria a colgajo TRAM libre.

\section{Obesidad}

La consideramos una contraindicación relativa, ya que pese a la mayor incidencia de necrosis grasa que se describe en estas pacientes, nosotros somos partidarios de realizar ajustes en el tamaño del colgajo y en su disposición respecto al pedículo, no observando así necrosis completas del colgajo.

Para otros autores (6), la necrosis grasa y la congestión venosa no se relacionan con el tabaquismo, edad de la paciente u otros factores, y sí estarían vinculadas en mayor medida con el sobrepeso de la paciente.

Nuestra experiencia contempla una media de edad de 50 años en nuestras pacientes, con un índice de masa corporal medio de $26^{\prime} 9 \mathrm{Kg} / \mathrm{m}^{2}$. Así pues nuestras pacientes son relativamente jóvenes con un sobrepeso bastante discreto. Como hemos publicado recientemente (7), el grosor medio del panículo adiposo en nuestros casos se encuentra en un rango de $3,3+/-1,2 \mathrm{~cm}$.

El ajuste del tamaño del colgajo al que hacemos referencia y la disposición del pedículo viene esquematizado en las figuras adjuntas. Considerando las dos filas de perforantes en cada hemiabdomen, con el diseño prequirúrgico del colgajo (Fig.5), es posible 


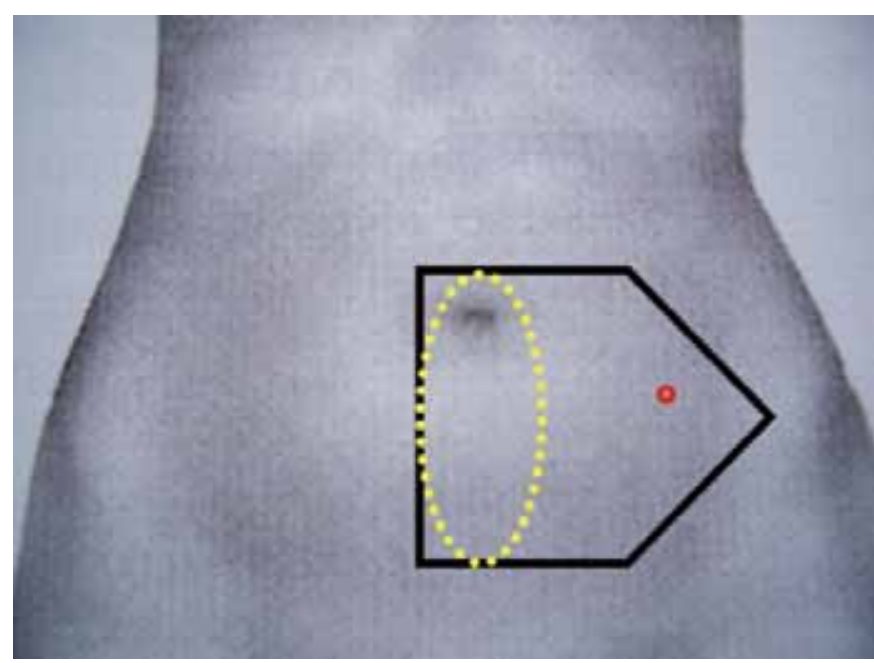

Fig. 6. Si el pedículo de mayor calbire se localiza en una situación excéntrica respecto al diseño original, situándose en el cuadrante III de Hartrampf, serían probables las carencias vasculares en los cuadrantes más distales.

que durante la disección del mismo localicemos el pedículo vascular óptimo en una situación extrínseca respecto al diseño primario, pese a que las pruebas prequirúrgicas nos hubiesen marcado vascularización adecuada a nivel medial o periumbilical; al elegir éste como el principal del colgajo, los territorios que teóricamente estarían irrigados directamente por perforantes (cuadrante I de Hartrampf) o por vasos axiales (cuadrante II de Hartrampf) podrían sufrir carencias vasculares (Fig.6), más relevantes en pacientes obesas. Por ello optamos por realizar intraoperatoriamente el "rediseño" comentado del colgajo, para conseguir así centralizar la perforante de mayor capacidad vascular (Fig.7).

Este pequeño refinamiento quirúrgico cobra más importancia en pacientes cuya obesidad podría entenderse como una contraindicación quirúrgica.

\section{Volumen mamario contralateral}

Entendido más como una controversia que como una contraindicación, nos referimos al volumen mamario para explicar nuestra metodología de trabajo en pacientes con hipertrofia mamaria contralateral.

En estas pacientes optamos por realizar una remodelación de la mama contralateral en un segundo tiempo quirúrgico, adaptando la mama al nuevo volumen y forma del colgajo, logrando así una máxima simetría. Mediante esta estructura de trabajo hemos alcanzado un alto nivel de satisfacción (93'9\%) entre nuestras pacientes (1). En este segundo tiempo, es cuando también realizamos la reconstrucción del complejo teloareolar de la mama reconstruida, mediante colgajos locales para el pezón, e injerto de espesor total o tatuaje para la areola (en la actualidad realizamos todos los casos mediante tatuaje).

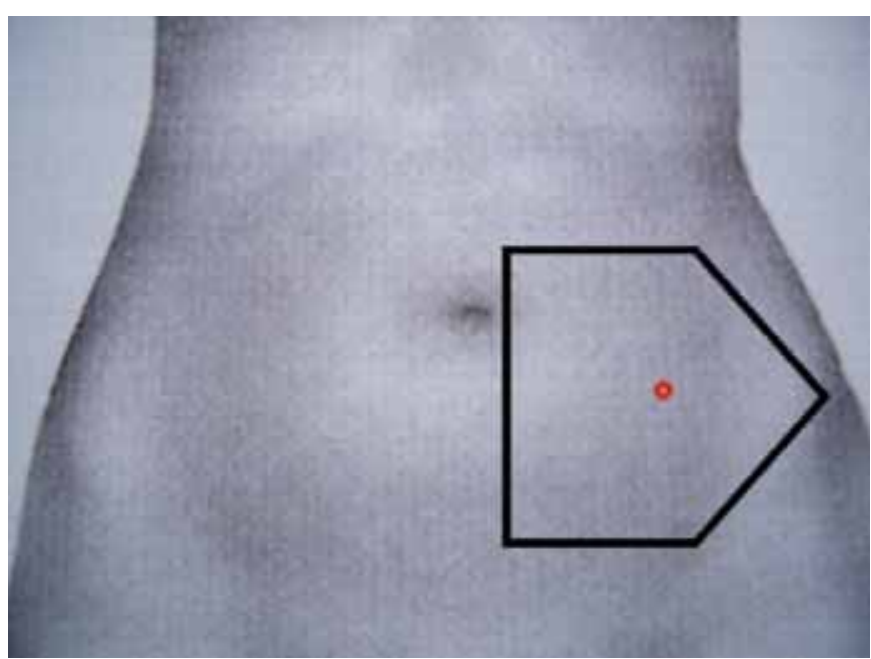

Fig. 7. Realizamos un rediseño intraoperatorio del colgajo de cara a centralizar el pedículo.

En casos de grandes volúmenes mamarios (>1000 cc) en los que la paciente rechaza una intervención sobre la mama sana, deberíamos considerar la reconstrucción mamaria mediante colgajo TRAM libre, ya que en estas pacientes se incrementa el riesgo de carencias en la perfusión vascular. Pese a esta consideración, deberíamos aclarar que el colgajo DIEP ha demostrado una adecuada perfusión, basándose en 1 (<750 gr) ó 2 (>750 gr) perforantes.

\section{Radioterapia}

Los efectos de la radioterapia sobre los vasos receptores estarían concretados por estos tres efectos nocivos: fibrosis perivascular, sufrimiento endotelial y trombosis microvascular. Pero se debe señalar que dichas consecuencias tienen un efecto dosis-dependiente, ya que pese a observar repercusiones venosas a dosis de 60-70 Grays (disección de la íntima, hialinosis...), bajando a dosis fraccionadas de 40-50 Grays (tendencia actual en el tratamiento del cáncer de mama) no observamos estos cambios. Así conseguimos que el tratamiento radioterápico previo a la reconstrucción no suponga una contraindicación para el empleo del colgajo DIEP.

La radioterapia tras reconstrucción mamaria inmediata aumenta el riesgo de necrosis grasa del colgajo, por lo que si presuponemos la posibilidad de este tratamiento adyuvante es preferible demorar la reconstrucción 6 meses.

Dado que nuestro Servicio se encuentra integrado en la Unidad de Patología Mamaria del Hospital Reina Sofía, contamos con la ventaja de poder realizar una estadificación anatomopatológica intraoperatoria, lo cual nos permite conocer en el acto quirúrgico de la mastectomía, la necesidad de radioterapia adyuvante. Esto viene determinado por la implantación, tras un proceso de validación de los diferentes 
especialistas de los Servicios integrantes de la Unidad, de la técnica de ganglio centinela (cuyas contraindicaciones aparecen reflejadas en la Tabla I). Por ello podemos realizar una contraindicación de reconstrucción inmediata en el mismo quirófano, sin necesidad de tener que aventurarnos en nuestras decisiones.

Con estas consideraciones, la actitud que proponemos respecto a este factor precisa de una comunicación fluida entre los Servicios integrantes de la Unidad de mama de cada centro.

\section{Gasto sanitario}

En la actualidad estamos recopilando los datos económicos relativos a nuestra propia casuística, labor que consideramos ardua puesto que los registros hospitalarios en este sentido no son lo precisos que desearíamos. Es por esto que, mientras dispongamos de estos datos con los que pretendemos hacer una futura publicación detallada, aplicamos a nuestra experiencia diaria los resultados publicados por otros autores en la bibliografía internacional.

Sin ánimo de querer entrar en una discusión entre la rentabilidad de la reconstrucción mamaria mediante tejidos autólogos o mediante prótesis (no solo en términos económicos sino también de resultados), sobradamente discutida en la literatura, nosotros enfocamos nuestro análisis a comparar los distintos colgajos más empleados y la rentabilidad del momento elegido para la reconstrucción. Es decir, nos planteamos la duda de si es más rentable realmente la reconstrucción inmediata respecto a la diferida (siendo indudable el valor psicológico que reporta a la paciente).

En nuestro caso, no realizamos reconstrucción inmediata mediante colgajo DIEP, empleando técnicas mediante prótesis en pacientes tributarias de mastectomía simple (prótesis expansoras, en la mayoría de los casos, o prótesis definitivas), o de remodelación oncoplástica mamaria en aquellas en las que se indica tumorectomía o cuandrantectomía.

Conocemos estudios como el de Kroll (8) que hacen referencia a que la reconstrucción inmediata mediante colgajo libre TRAM o DIEP es incluso más cara que la reconstrucción diferida. Recientemente un completo estudio del grupo de trabajo de Fu-Chan Wei (9) ha constatado la ausencia de diferencias estadísticamente significativas en los costes y en las complicaciones entre ambos tiempos reconstructivos, hasta el punto de afirmar que los costes no deberían ser un factor determinante a la hora de decidir el momento de la reconstrucción, ofreciendo interesantes resultados que no alcanzan la significación estadística necesaria $\left(\mathrm{p}=0^{\prime} 345\right)$ para demostrar que un método es económicamente más rentable que el otro.
Tabla I. Contraindicaciones para ganglio centinela

- Tamaño tumoral superior a $3 \mathrm{~cm}$.

- Multifocalidad.

- Signos de afectación tumoral axilar.

- Quimioterapia o cirugía axilar previas.

- Embarazo.

Si comparamos el empleo de DIEP frente a reconstrucción mediante otros colgajos también habituales en la práctica diaria, el colgajo DIEP no parece suponer un incremento en el gasto. Comparando el colgajo DIEP con el TRAM libre en términos de gasto sanitario, diversas publicaciones (10) defienden la opción del colgajo de perforantes, llegando a señalar diferencias superiores a los 1345 dólares, reflejados sobre todo en gastos de quirófano. Si lo comparamos frente al colgajo de dorsal ancho, otro reciente estudio (11), no encuentra diferencias estadísticamente significativas ( $\left(\mathrm{p}>00^{\prime} 05\right)$ entre los días de ingreso hospitalario de las pacientes reconstruidas con uno u otro método (no más de 8 días de media en ambos casos), pero sí describe un ahorro del gasto estadísticamente significativo $\left(\mathrm{p}<0^{\prime} 01\right)$ comparando la carga farmacológica postoperatoria de las pacientes, siendo el gasto menor en el caso de aquellas reconstruidas mediante colgajo abdominal.

\section{Conclusiones}

El colgajo DIEP se ha consolidado como uno de los métodos más relevantes en reconstrucción mamaria y las contraindicaciones que se plantean a la hora de decidirnos por él en vez de por otras opciones, son aún hoy factores muy controvertidos. Estas características no deberían condicionar su veto como opción terapéutica, siendo precisa una individualización del caso y un estudio detenido de los condicionantes de cada paciente.

\section{Dirección del autor}

Dr. César Casado Sánchez

Hospital Universitario Reina Sofía.

Hospital Provincial.

Servicio de Cirugía Plástica y Reparadora.

Avda. Menéndez Pidal s/n. 14004, Córdoba. España e-mail: doctorcasado@gmail.com

Bibliografía

1. Cabrera, E; Redondo, A; Dean, A., et al.: "Satisfacción en pacientes con reconstrucción mamaria con colgajo DIEP”. Cir. plást. iberolatinoam. 2006, 32(3): 169.

2. Craigie J.E., Allen R.J., DellaCroce F.J., et al.: "Autogenous breast reconstruction with the deep inferior epigastric perforator flap". Clin Plastic Surg 2003; 30: 359. 
3. Nahabedian, M.Y., Momen, B., Manson, P.N.: "Factors Associated with Anastomotic Failure after Microvascular Reconstruction of the Breast". Plast Recons Surg. 2004, 114(1):74.

4. Chang, D. W., Reece, G. P., Wang, B., et al.: "Effect of smoking on complication in patients undergoing free TRAM breast reconstruction”. Plast Recons Surg. 2000, 105: 2374.

5. Padubidri, A. N., Yetman, R., Browne, E., et al.: "Complications of postmastectomy breast reconstruction in smokers, ex-smokers, and nonsmokers". Plast Recons Surg. 2001, 107: 342.

6. Nahabedian M.Y., Momen, B., Galdino, G., et al.: "Breast Reconstruction with the Free TRAM or DIEP Flap: Patient Selection, Choice of Flap, and Outcome". Plast Recons Surg. 2002, 110(2):466

7. Fidalgo F., Redondo A., Dean A., et al: "Remodelación de los colgajos TRAM libres y DIEP”. Cir. plást. iberolatinoam. 2007, 33: 1.
8. Kroll, S.S., Reece, G.P., Miller, M.J., et al.: "Comparison of cost for DIEP and free TRAM flap breast reconstructions". Plast Recons Surg. 2001, 107: 1413.

9. Cheng, M., Lin, J., Ulusal, B.G., et al.: "Comparisons of Resource Costs and Success Rates between Immediate and Delayed Breast Reconstruction Using DIEP or SIEA Flaps under a Well-Controlled Clinical Trial”. Plast Recons Surg. 2006, 117(7):2139.

10. Thoma, A., Veltri, K., Khuthaila, D., et al.: "Comparison of the Deep Inferior Epigastric Perforator Flap and Free Transverse Rectus Abdominis Myocutaneous Flap in Postmastectomy Reconstruction: A Cost-Effectiveness Analysis". Plast Recons Surg. 2004, 113(6): 1650 .

11. Misra, A., Chester, D., Park, A.: "A Comparison of Postoperative Pain between DIEP and Extended Latissimus Dorsi Flaps in Breast Reconstruction". Plast Recons Surg. 2006, 117(4), 1108. 


\title{
Comentario al trabajo uAnálisis de controversias en reconstrucción mamaria con colgajo DIEPII
}

\author{
Dra. Martina Marín Gutzke \\ Cirujano Plástico. Servicio de Cirugía Plástica Hospital Ramón y Cajal. Madrid. España.
}

Los Drs. Casado Sánchez, Cabrera Sánchez, Redondo Camacho y Rioja Torrejón presentan el trabajo "Análisis de controversias en reconstrucción mamaria con colgajo DIEP", desarrollado en un Servicio de Cirugía Plástica que ha demostrado su experiencia en reconstrucción mamaria autóloga tanto con el colgajo TRAM libre como con el DIEP, con series ya publicadas en esta revista (1-3).

El planteamiento de controversias constituye una herramienta metodológica útil al definir y analizar los elementos de debate y es, a su vez, un formato de difícil desarrollo. Se agradece a los autores el esfuerzo realizado en este sentido.

En el presente trabajo se describe el impacto de ciertos factores, entre otros el tabaquismo, el tratamiento radioterápico, la historia de intervenciones quirúrgicas previas en la región abdominal, el grado de obesidad y la magnitud del gasto sanitario. A excepción del gasto sanitario, estos factores pueden ser contemplados como factores de riesgo más que como elementos de controversia. El último punto de debate, el gasto sanitario, no ha sido cuantificado. La metodología desarrollada en el trabajo es descriptiva y no analítica, lo cual no permite elaborar conclusiones o definir indicaciones y contraindicaciones. La recomendación por parte de los autores del colgajo DIEP sobre otras opciones terapéuticas se expone sin justificar suficientemente los elementos del debate científico sobre este tema.

Dado que los autores posen una experiencia demostrada en la reconstrucción mamaria mediante colgajos libres, un estudio comparativo entre el TRAM libre y el DIEP puede ser de gran interés en un tema de controversia actual, sobre todo al considerar las variedades de preservación muscular del TRAM libre (MS1, MS2), teniendo en cuenta la complejidad de la disección, la perfusión del colgajo y la morbilidad de la pared abdominal (4-6). Por último, el debate se dirige hacia la planificación preoperatoria mediante los sistemas de detección de perforantes y planificación preoperatoria de los colgajos (7).

\section{Bibliografía}

1. Rioja L, Redondo A, García E, Soto R, Fidalgo F, Torres J, Haro J.: "Reconstrucción mamaria con colgajo TRAM libre". Cir plást iberolatinoam 2002; 28: 39.

2. Cabrera E, Redondo A, Dean A, Benítez J, Torre C, De Piero G, Navarro J, Molina H, Ramírez J, Rioja L.: "Satisfacción en pacientes con reconstrucción mamaria con colgajo DIEP". Cir plást iberolatinoam 2006, 32(3): 169.

3. Fidalgo F, Redondo A, Dean A, Rioja L.: "Remodelación de los colgajos TRAM libres y DIEP”. Cir plást iberolatinoam 2007, 33: 37.

4. Nahabedian MY, Momen B, Galdino G, Manson PN.: "Breast reconstruction with the free TRAM or DIEP flap: patient selection, choice of flap, and outcome." Plast Reconstr Surg 2002 110: 466.

5. Nahabedian MY, Tsangaris T, Momen B.: "Breast reconstruction with the DIEP Flap or the Muscle-Sparing (MS-2) Free TRAM flap: Is there a difference?" Plast Reconstr Surg 2005, 115: 436.

6. Bajaj AK, Chevray PM, Chang DW.: "Comparison of donor-site complications and functional outcomes in free muscle-sparing TRAM flap and free DIEP flap breast reconstruction". Plast Reconstr Surg 2006, 117: 737.

7. Masiá, J, Clavero JA, Carrera A.: "Planificación preoperatoria de los colgajos de perforantes”. Cir plást iberolatinoam 2006, 32: 237.

\section{Respuesta al comentario de la Dra. M. Marín Gutzke}

\section{Dr. César Casado Sánchez}

Agradezco sinceramente en nombre de mis compañeros, y en el mío propio, los comentarios de la Dra. Marín Gutzke, cuya trayectoria en reconstrucción microquirúrgica avala sus observaciones.

Nos propusimos mostrar nuestra experiencia en reconstrucción mamaria mediante colgajo DIEP, desde la perspectiva de una correcta indicación del colgajo acorde a las características de cada paciente, muchas veces motivo de discusión. No pretendemos en ningún caso ofrecer una guía de buena praxis, dado que efectivamente abordamos esta materia desde un punto de vista descriptivo, pero nos apoyamos desde nuestros inicios en la experiencia de otros grupos con una trayectoria más dilatada, para ofrecer a nuestras pacientes resultados adecuados dentro de nuestras capacidades. Con el paso de los años, la evolución del colgajo y sus indicaciones, nos hemos ido adaptando a la realidad del mismo, y se ha expuesto en el artículo nuestra actual metodología, que nos ha llevado a conseguir los resultados referidos en las diversas publicaciones al respecto.

Respecto a la interesante observación realizada acerca de las par- ticularidades del DIEP comparado con el colgajo TRAM libre MS2, venimos observando que las repercusiones en términos de morbilidad en la pared abdominal de ambos colgajos es similar (de forma igualmente meramente descriptiva). Nos parecen acertadas todas aquellas medidas que faciliten la disección del colgajo sin repercusión sobre la viabilidad del mismo, limiten los riesgos inherentes a la manipulación del pedículo, considerando unas secuelas mínimas a nivel del territorio donante.

Son indiscutibles las ventajas que aportan las recientes técnicas de planificación de los colgajos microvascularizados, mereciendo los impulsores de estás medidas nuestro reconocimiento. No ha sido hasta fechas recientes cuando disponemos en nuestro centro del apoyo necesario para su desarrollo. Por ello, continuamos empleando de forma mayoritaria en el desarrollo de colgajos de perforantes el Doppler de ultrasonido, siendo fundamental la confirmación intraoperatoria de las adecuadas características del pedículo seleccionado inicialmente. 